\title{
Random Harmonic Detection and Compensation Based on Synchronous Reference Frame
}

\author{
Yanbo Che, Zhaojing Yin, Shuyan Yu, and Qiang Sun \\ Key Laboratory of Smart Grid of Ministry of Education, Tianjin University, Tianjin 300072, China \\ Correspondence should be addressed to Yanbo Che; ybche@tju.edu.cn
}

Received 16 January 2017; Revised 11 May 2017; Accepted 14 May 2017; Published 6 June 2017

Academic Editor: Ephraim Suhir

Copyright ( 2017 Yanbo Che et al. This is an open access article distributed under the Creative Commons Attribution License, which permits unrestricted use, distribution, and reproduction in any medium, provided the original work is properly cited.

\begin{abstract}
Algorithms for harmonic detection and compensation are important guarantees for an active power filter (APF) to achieve the harmonic control function and directly determine the overall performance. Existing algorithms usually need a large amount of computation, and the compensation effect of specified order harmonic is also limited. DC side capacitor voltage at sudden change of load is affected by the algorithm as well. This paper proposes a new algorithm for harmonic detection and compensation based on synchronous reference frame (SRF), in which a band-pass filter with center frequency of $6 k$ th harmonic is designed in fundamental frequency SRF to extract random harmonic current with two different frequencies of $(6 k \pm 1)$ th harmonic in stationary reference frame. This new algorithm can rapidly detect any specified harmonic, and it can adjust the power factor to compensate reactive power. Meanwhile, it has few impacts on DC side capacitor voltage under complicated operating conditions such as sudden change of load. The correctness and effectiveness of this new algorithm are verified by simulation and experiment.
\end{abstract}

\section{Introduction}

With the large-scale application of power electronics devices in recent years, the amount of various nonlinear loads has been increased rapidly. Current waveform distortion and harmonic problem is becoming more and more serious; hence the research and application of harmonic control devices have become a hot research topic at home and abroad [1-3]. At present, there are two main types of filter, passive power filter (PPF) and active power filter (APF). PPF removes harmonic or prevents harmonic spreading by adding extra impedance branches to a system. PPF is easy to be resonant with the power grid, and its compensation characteristic is easy to be affected by the impedance of the power network. The compensation accuracy is low and the flexibility of its application is poor. These drawbacks limit its application in some occasions with high requirements [4]. Adopting high-performance main circuit of power electronic converter and control system, APF can quickly detect changes in harmonic currents, then compensate harmonic, and adjust power quality according to different compensation targets.
Being better than PPF in both compensation precision and flexibility of harmonic current detection, APF has gradually got more attention and research [5-8].

The detection and compensation performance of an APF is mainly related to the extraction algorithm of the system compensation current and the control strategy of the main power inverter. The performance of a compensation current extraction algorithm largely depends on the accuracy, fastness, and real-time of the current extraction. The key to controlling a main power inverter is to ensure a fast tracking of instruction current by APF output current and keep their consistency. Therefore, it is an important issue improving the accuracy of APF harmonic compensation to catch the instantaneous harmonic and reactive components from the power grid currents quickly and accurately. Meanwhile, with the rapid development of large-scale integrated circuit, especially digital signal processor technology, the technology of extracting harmonic and reactive current in APF system has gradually developed from analog to digital form, which greatly improves the speed of current extraction and the accuracy of compensation [9]. However, the traditional 
TABLE 1: Comparison of different methods.

\begin{tabular}{|c|c|c|c|c|c|c|}
\hline & Filter & Wavelet Transform & Neural Network & FFT & PQ & SRF \\
\hline Computation & A & $\mathrm{D}$ & $\mathrm{C}$ & $\mathrm{E}$ & B & B \\
\hline Response speed & E & $\mathrm{D}$ & $\mathrm{B}$ & $\mathrm{C}$ & B & A \\
\hline Reliability & $\mathrm{E}$ & $\mathrm{D}$ & $\mathrm{C}$ & $\mathrm{D}$ & B & A \\
\hline Compensation precision & $\mathrm{E}$ & $\mathrm{D}$ & $\mathrm{C}$ & A & $\mathrm{B}$ & A \\
\hline Ability of random harmonic detection and compensation & No & No & No & Yes & No & No \\
\hline
\end{tabular}

harmonic detection and compensation methods which detect and compensate the content of harmonic cannot meet the functional requirements on variety to APF [10-12]. It is urgent to search a method that could detect the random harmonic current or voltage in load and then compensate them according to actual demands.

At present, the main methods for harmonic detection and compensation are classified as Filter method [13], Wavelet Transform method [14], Neural Network method [15, 16], FFT method [17, 18], PQ method [19], and SRF method [20]. The authors have deeply studied these methods by reading a large number of literatures [21-27] and evaluated them from computation, response speed, reliability, and compensation precision. As shown in Table 1, A to $\mathrm{E}$ stand for the grades from highest to lowest, and their abilities of random harmonic detection and compensation are also compared. This table indicates that the Filter method, Wavelet Transform method, Neural Network method, and FFT method only are suitable for some special occasions due to their shortcomings. Although the PQ method and the traditional SRF method, which are based on Instantaneous Reactive Power Theory [28], show good performance in all aspects and are widely used, they lack the function of detecting and compensating for random harmonics.

Different from traditional harmonic detection algorithm, random harmonic detection algorithm can detect and compensate specified order harmonic, improve processing speed, reduce work load, and thus get attention by scholars. Taking a three-phase three-wire parallel APF as research representative, this paper proposes a new SRF-based algorithm for random harmonic current detection and compensation. It can detect and compensate the specified harmonic, reduce the computation amounts, and have few impacts on DC side capacitor voltage when the loads mutate. Finally, simulation and experiment show the effectiveness and feasibility of this algorithm.

\section{Basic Principle}

2.1. Basic Principle of APF. The concept of APF was first proposed by Japanese scientists in the early 70s. The basic principle of APF is to generate a current that has the same amplitude and opposite phase with harmonic current by controllable power semiconductor devices and inject it into the grid, intentionally to cancel the total harmonic current and compensate in real-time. According to different applications, the active power filter can be divided into two categories: DC active power filter and AC active power filter.

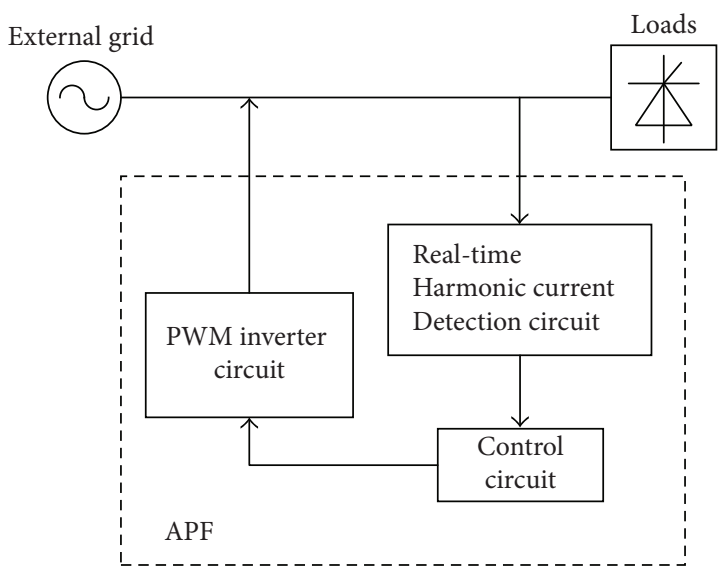

FIGURE 1: Schematic diagram of APF.

The former is mainly applied to HVDC system, and the latter (often cited as APF will be discussed in this paper) to AC power system of different voltage levels.

An APF usually consists of two functional parts, detecting harmonic signal and generating compensating component. As shown in Figure 1, distorted proportion of the current and voltage in grid are detected by the detection circuit of APF. Corresponding compensation current component is then generated from the power circuit under certain control strategy and injected into the grid to achieve the purpose of harmonic elimination.

\subsection{Basic Idea of SRF-Based Harmonic Detection Algorithm.} The SRF-based algorithm works by changing a fundamental positive sequence current into direct current by Park Transform, then filtering out the harmonic and reactive current through a low-pass filter (LPF), and finally getting the compensation current reference value [16], as shown in Figure 2.

Current in nonlinear load such as common rectifier bridge consists of the fundamental and $(6 n \pm 1)$ th harmonic components, respectively, corresponding to direct current (DC) and alternating current (AC) components in stationary reference frame when converted into fundamental frequency SRF. A LPF precisely designed in fundamental frequency SRF can greatly improve the percentage of fundamental component, and then the harmonic component can be obtained by subtracting the fundamental harmonic from the load waveform. 


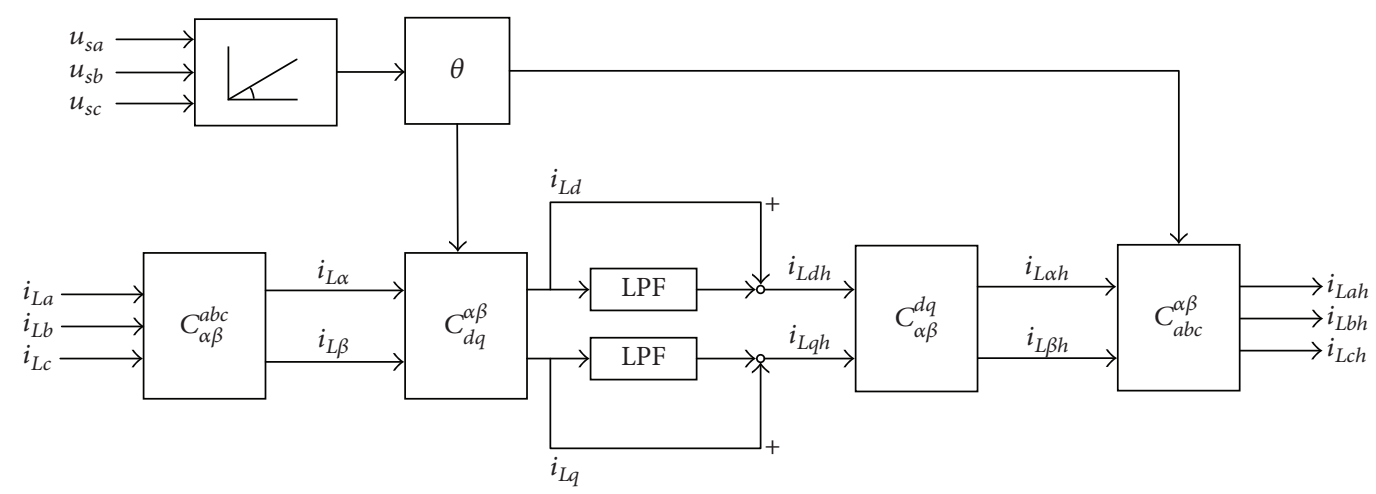

FIGURE 2: The basic principle of SRF.

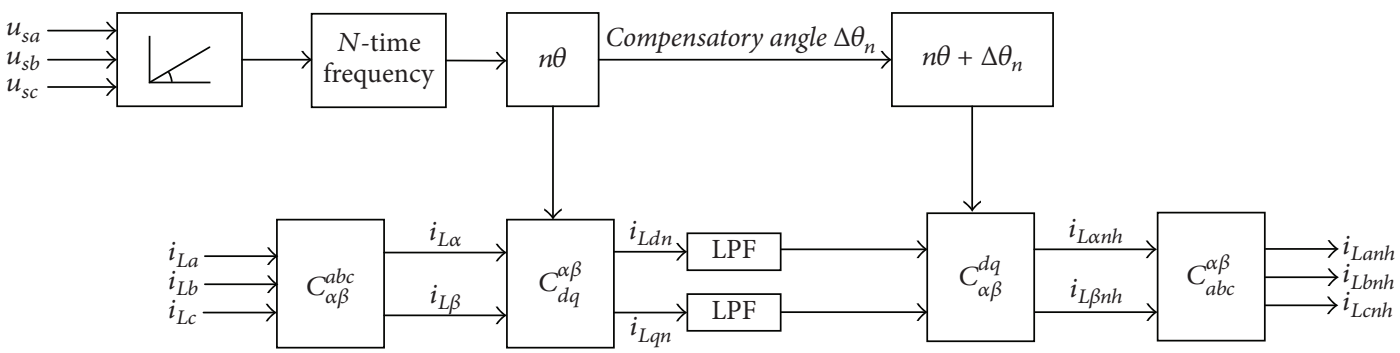

FIGURE 3: The basic principle of SRF with compensatory angle.

\section{SRF-Based Random Harmonic Detection and Compensation}

3.1. Stability of DC Side Capacitor Voltage. Stabling the DC side capacitor voltage is an important premise to effectively compensate the harmonic currents generated by load. The basic idea (of stabling the DC side capacitor voltage) is to absorb the fundamental active power in the grid by treating the active power filter as the current source device. The lowpass filter in the traditional harmonic detection algorithm is carried out in fundamental frequency SRF. When the load changes suddenly, the time delay during hall current detection of load side is usually neglected. There is a time delay in the harmonic compensating reference calculation process of LPF to extract the current, so the LPF is unable to trace the changes in input. If the load changes suddenly during the process of harmonics compensating, although the APF can instantly sense this change, some fundamental components will still be remained in harmonic detection algorithm. So for the time period from the beginning of mutation in load to the end of time delay in LPF, APF actually absorbs active power from the grid. These active components raise the DC side capacitor voltage, and stronger load current or change causes more obvious fluctuation in the DC side capacitor voltage.

The harmonic compensating reference in the random harmonic detection algorithm does not come from the result of subtracting the LPF output from the load current, so the compensating reference, when load changes do not contain fundamental component that largely fluctuate the DC side capacitor voltage, and the voltage can be easily maintained.
3.2. Random Harmonic Detection and Compensation. As stated above, when the load suddenly changes, the influence from random harmonic detection and compensation device on the DC side capacitor voltage is much smaller than that from a full compensation shown in Figure 2. The random harmonic detection method with preset compensatory angle is commonly used and given in Figure 3.

The application of the random harmonic detection algorithm in Figure 3, however, is limited by large computation. This algorithm will be updated in this paper to reduce computation load and realize quick random detection and compensation of harmonics. The updated algorithm can also ensure the stability of DC side capacitor voltage.

In two-phase stationary reference frame, the vector of the load current is composed of the vectors of fundamental and harmonic currents. The $(6 n+1)$ th harmonic current vectors are in the same direction with the fundamental current vector and are defined as positive sequence components; correspondingly, the $(6 n-1)$ th harmonic current vectors are in the opposite direction to the fundamental current vector and are defined as negative sequence components. The rotational angular velocity of a pair of adjacent harmonic current of positive and negative sequences relative to the fundamental vector is $6 n \omega$ in two-phase stationary reference frame; therefore, the fundamental, positive, and negative sequences of the load currents under a stationary reference frame can be, respectively, described as follows:

$$
\begin{aligned}
i_{L \alpha 1} & =\sqrt{\frac{3}{2}} I_{1} \sin (\omega t), \\
i_{L \beta} & =-\frac{\sqrt{3}}{2} I_{1} \cos (\omega t),
\end{aligned}
$$




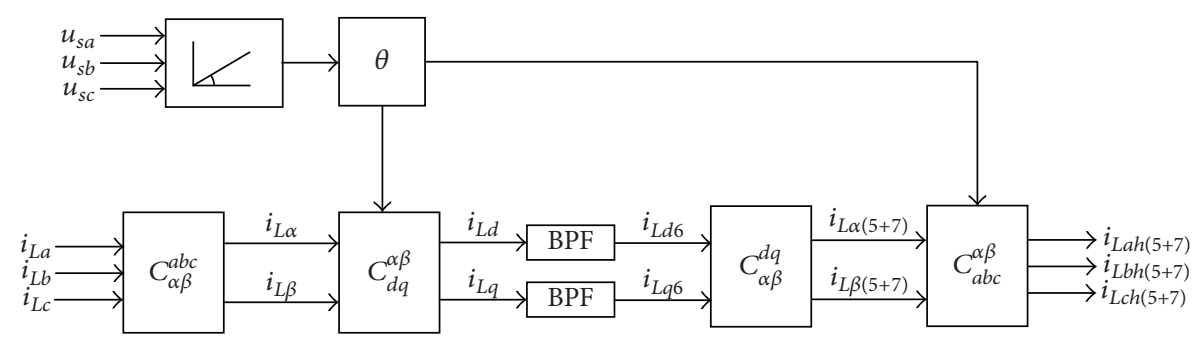

FIGURE 4: The schematic diagram of new SRF-based algorithm for harmonic detection.

$$
\begin{aligned}
& i_{L \alpha h}^{+}=\sqrt{\frac{3}{2}} I_{n} \sin (n \omega t), \quad n=6 k+1, k=1,2, \ldots, \\
& i_{L \beta h}^{+}=-\frac{\sqrt{3}}{2} I_{n} \cos (n \omega t), \quad n=6 k+1, k=1,2, \ldots, \\
& i_{L \alpha h}^{-}=\sqrt{\frac{3}{2}} I_{n} \sin (n \omega t), \quad n=6 k-1, k=1,2, \ldots, \\
& i_{L \beta h}^{-}=\frac{\sqrt{3}}{2} I_{n} \cos (n \omega t), \quad n=6 k-1, k=1,2, \ldots
\end{aligned}
$$

Transfer them with the expression in (2), and then fundamental and harmonic components in fundamental frequency SRF can be obtained, as shown in (3):

$$
\begin{aligned}
& {\left[\begin{array}{l}
i_{L d} \\
i_{L q}
\end{array}\right]=C_{d q}^{\alpha \beta}\left[\begin{array}{l}
i_{L \alpha} \\
i_{L \beta}
\end{array}\right]=\left[\begin{array}{cc}
\cos \theta & \sin \theta \\
-\sin \theta & \cos \theta
\end{array}\right]\left[\begin{array}{c}
i_{L \alpha} \\
i_{L \beta}
\end{array}\right],} \\
& i_{L d}=\sqrt{\frac{3}{2}}\left[I_{1}+\sum_{k=1,2, \ldots}\left(-I_{6 k-1}+I_{6 k+1}\right) \cos (6 k \omega t)\right], \\
& i_{L q}=\sqrt{\frac{3}{2}}\left[I_{1}+\sum_{k=1,2, \ldots}\left(I_{6 k-1}+I_{6 k+1}\right) \sin (6 k \omega t)\right],
\end{aligned}
$$

where $I_{1}$ is the amplitude value of the fundamental current and $I_{n}$ of the $n$th harmonic current. The physical meaning of (3) is that $(6 k \pm 1)$ th harmonics in stationary reference frame turn into $6 k$ th harmonic in fundamental frequency SRF.

The sum of adjacent positive and negative sequence harmonic current vectors in stationary reference frame is transferred into that of harmonic current vectors with the same rotational speed in fundamental frequency SRF. On the basis of SRF, two different harmonics $(6 k \pm 1)$ in stationary reference frame can be abstracted through a pair of band-pass filter (BPF) (6k) in fundamental frequency SRF. For example, the schematic diagram detecting 5 th harmonic negative and 7th harmonic positive sequence components of a rectifier load is shown in Figure 4, in which the center frequency of second-order BPF is $300 \mathrm{~Hz}$.

Extended from the SRF algorithm to detect harmonic, this new algorithm is able to control the power factor as well,
TABLE 2: Simulation parameters of all digital harmonic devices.

\begin{tabular}{lc}
\hline Parameters & Value \\
\hline Grid voltage & $0.4 \mathrm{KV}$ \\
DC side voltage & $0.9 \mathrm{KV}$ \\
DC side capacitor voltage & $6800 \mu \mathrm{F}$ \\
Output impedance & $0.6 \mathrm{mH}, 0.01 \Omega$ \\
Load resistance & $5 \Omega$ \\
\hline
\end{tabular}

so the reactive power compensation can be achieved. Meanwhile, the number of second-order BPFs and the computation of CPU are also reduced.

\section{Simulation Analysis}

4.1. Random Harmonic Detection and Compensation. From the above analysis, it is possible to detect harmonics by designing multigroup of BPFs with center frequencies of $6 k$, $k=1,2,3, \ldots$ in the fundamental frequency SRF. Each group of BPF can extract positive and negative harmonic sequence harmonics with different frequency at the same time.

To verify the correctness of the proposed algorithm, a model of harmonic suppression is built in MATLAB (Simulink), of which simulation parameters are shown in Table 2. Current waveform of the rectifier bridge with pure resistive load is shown in Figure 5.

The $d, q$ components of harmonic current with the order less than 31 are shown in Figures 6 and 7, respectively. The compensated current and its spectrum are given in Figure 8.

According to the current waveform and harmonic distortion rate after compensation, harmonics of all frequency are well compensated by the APF with the proposed method.

4.2. Waveform Comparison under Sudden Change of Load. For sudden changes in load, simulate and compare the effects of full compensation and random compensation. The nonlinear load consists of a bridge rectifier and a pure resistance, and the load current is suddenly doubled at $0.6 \mathrm{~s}$ and then halved at $0.75 \mathrm{~s}$, as shown in Figure 9.

Figures 10 and 11 show the corresponded grid currents for full compensation and random compensation, respectively. Figures 12 and 13 are waveforms for DC side capacitor voltages under these two kinds of compensations.

These simulation results indicate that the SRF-based algorithm for harmonic detection and compensation proposed in 


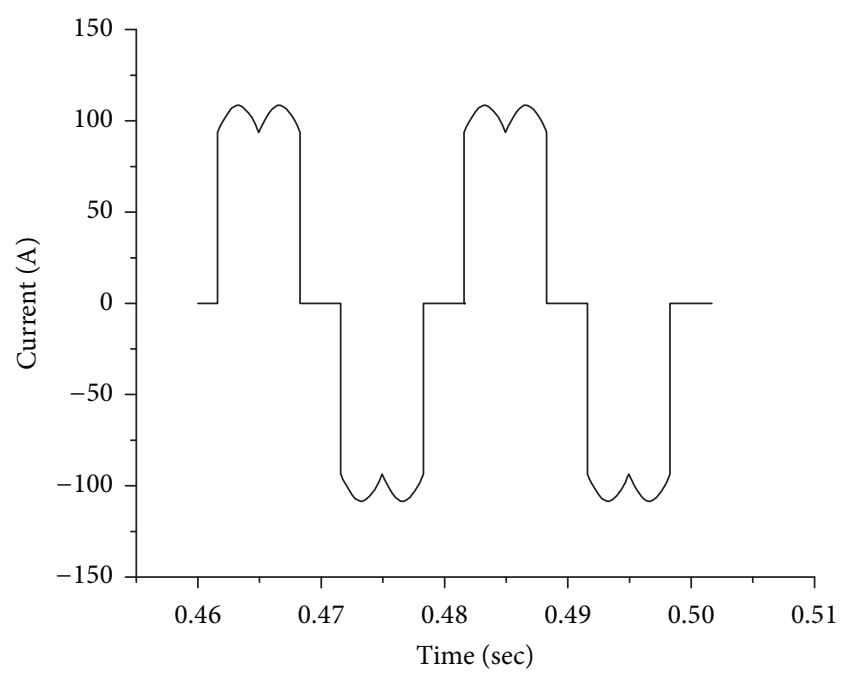

FIGURE 5: Current waveform of the rectifier bridge with pure resistive load.

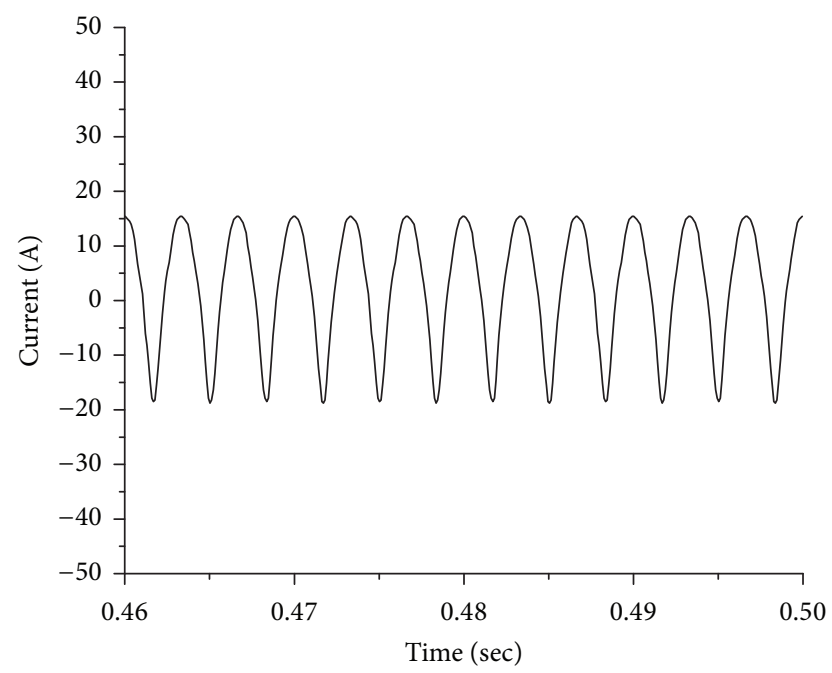

FIgURE 6: The $d$ component of harmonic current.

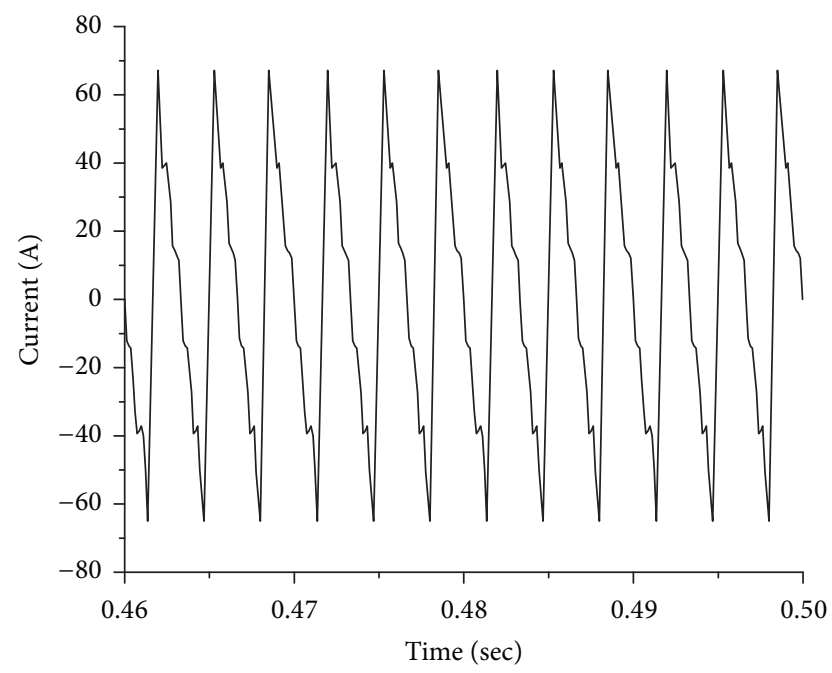

FIGURE 7: The $q$ component of harmonic current.
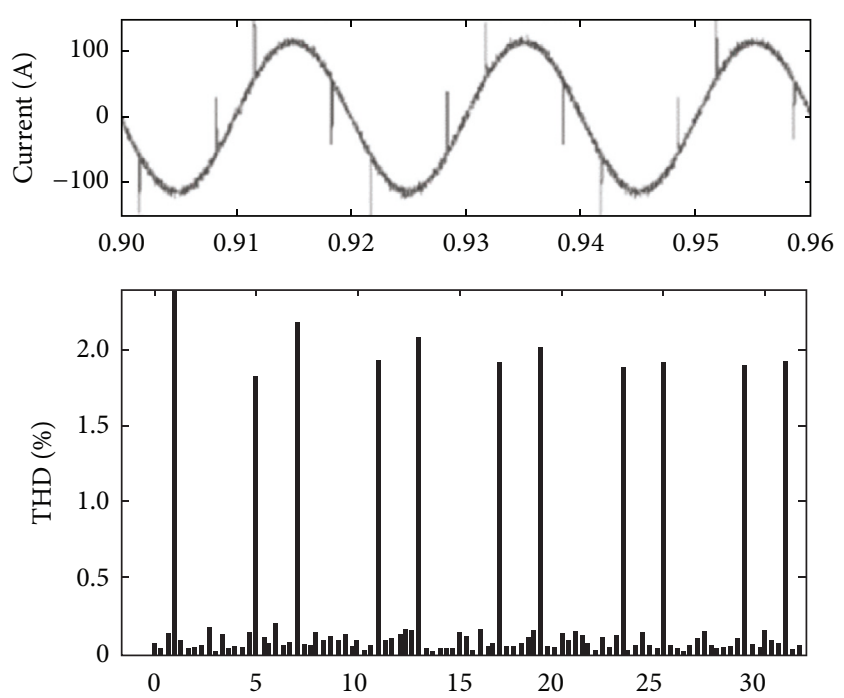

FIGURE 8: Compensated current waveform and spectral waveform.

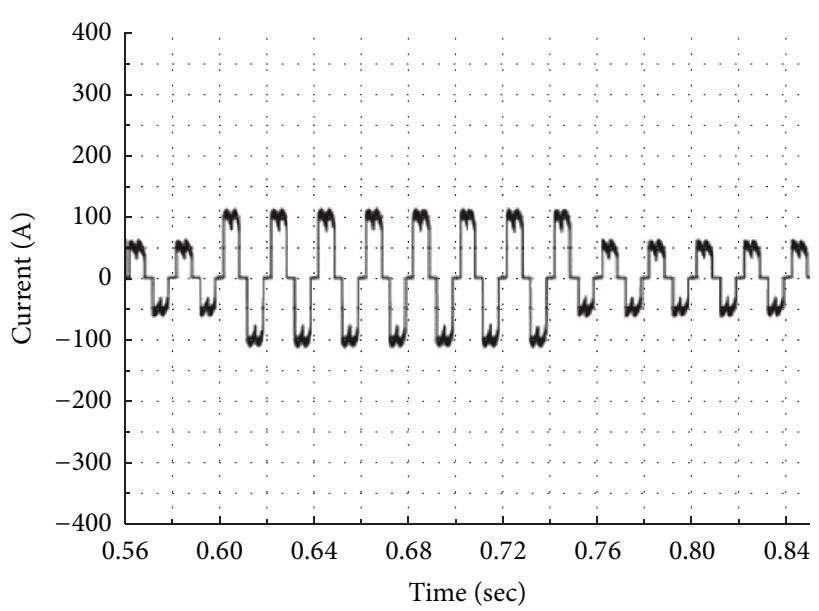

FIGURE 9: The load current waveform.

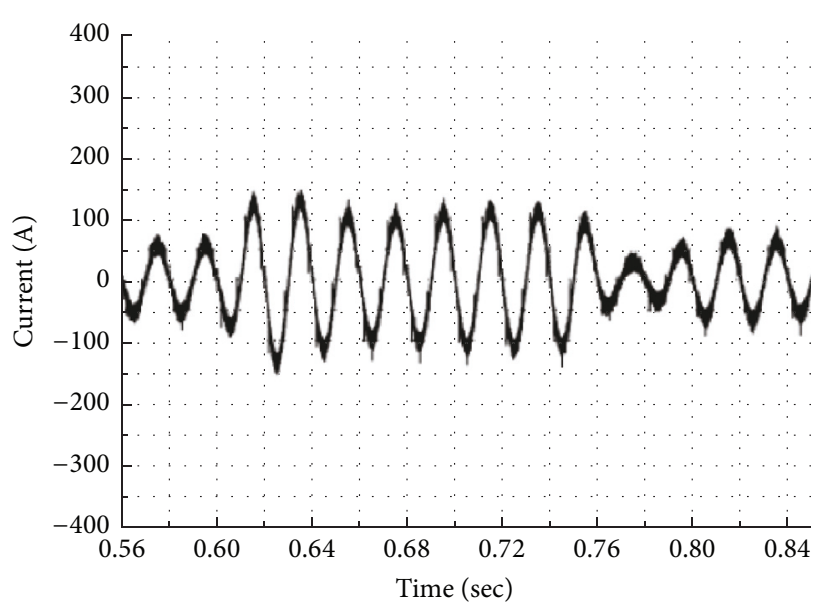

FIGURE 10: The grid current waveform under full compensation. 


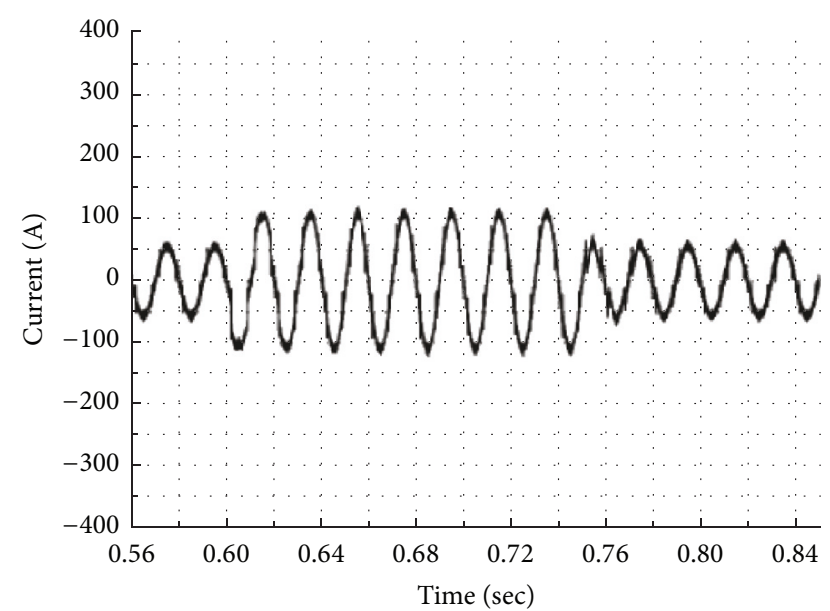

FIGURE 11: The grid current waveform under random compensation.

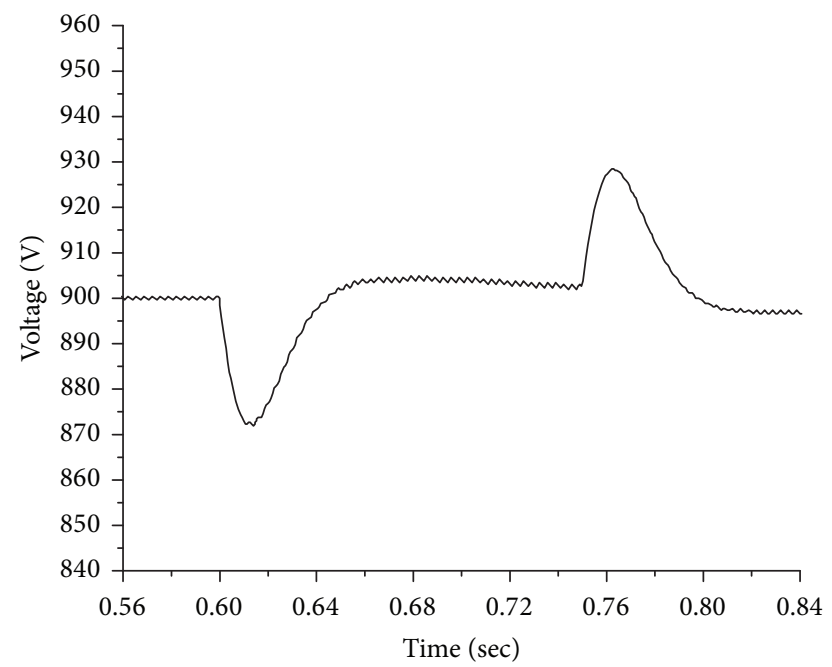

FIGURE 12: The capacitor voltage waveform under full compensation.

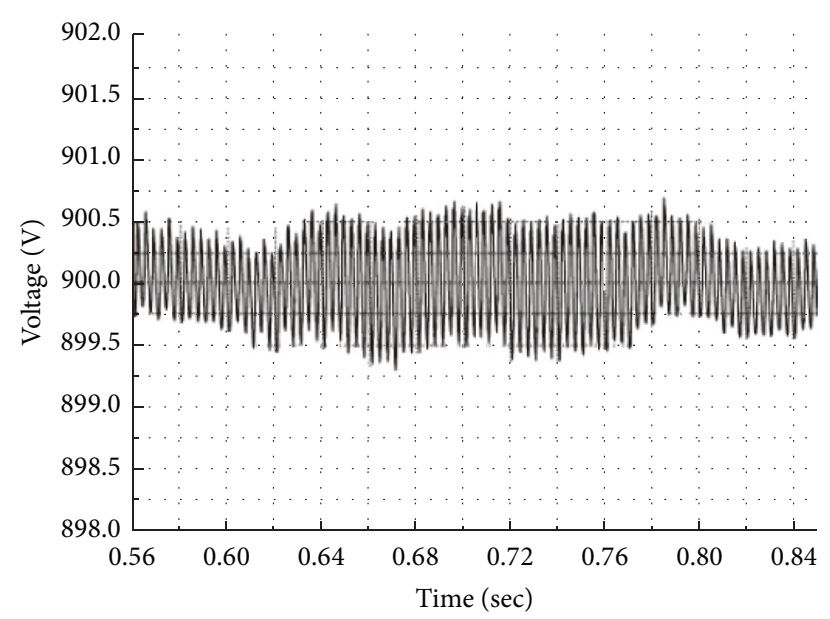

FIGURE 13: The capacitor voltage waveform under random compensation.

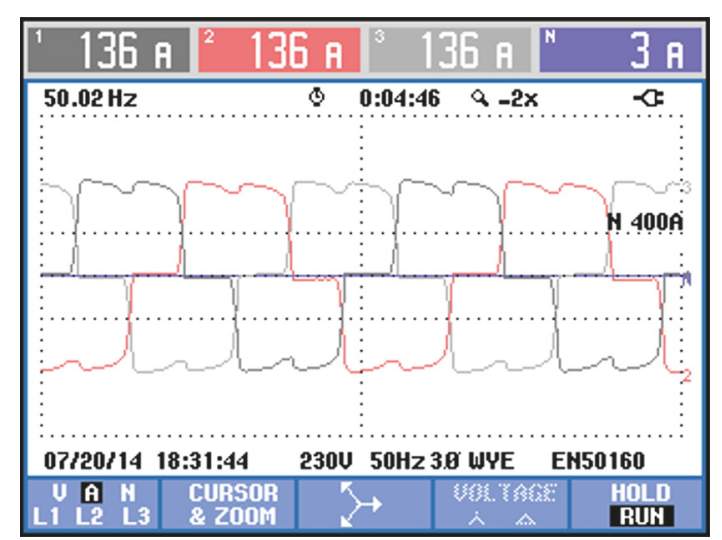

FIGURE 14: Current fluctuation curve before compensation.

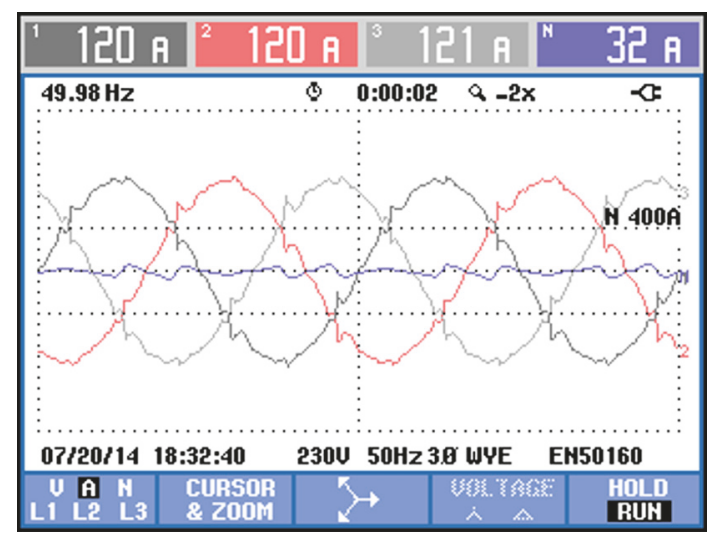

FIGURE 15: Current fluctuation curve after compensation.

this paper has good filtering effect to the harmonic current generated by simulation. When the load current changes suddenly, the random harmonic compensation algorithm does not import fundamental components, so it will not affect the DC side capacitor voltage.

\section{Experimental Verification}

The algorithm of random harmonic detection and compensation proposed in this paper is employed in a full digital controller for APF to verify its effect. The load is composed of a three-phase control rectifier and a pure resistance set to $3 \Omega$. A power quality analyzer of model FLUKE 434 was selected as measurement instrument. The current waveforms of three-phase grid before and after compensation are shown in Figures 14 and 15, respectively, and their harmonic contents are shown in Figures 16 and 17.

The heavily distorted current is restored to normal waveform after harmonic detection and compensation, and the effect of active power filter with our algorithm is significant. The experimental results show that the SRF-based method for harmonic detection and compensation can be well used to detect and compensate harmonics of specified order, and the DC side capacitor voltage can also be stable even when the 


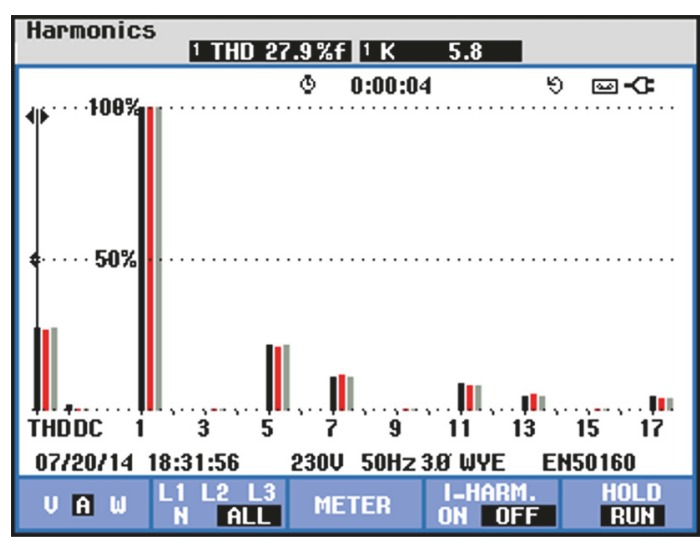

FIGURE 16: Current harmonic contents before compensation.

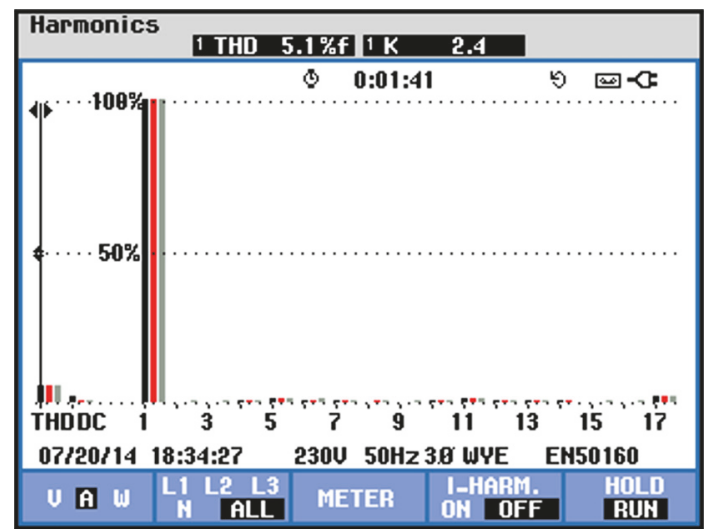

FIGURE 17: Current harmonic contents after compensation.

load changes suddenly. This is the key why the APF can filter efficiently.

\section{Conclusion}

The paper proposes a new SRF-based algorithm for random harmonic detection and compensation, and its mathematical principle is also deduced. Simulation and experiment show that the method is correct and practical. The conclusion can be drawn as follows.

Compared with traditional SRF harmonic detection algorithms, the computation is significantly reduced. When this new algorithm is applied to APF system, the operation burden of the digital controller can be reduced effectively and the control speed of the overall system is improved.

Avoiding obtaining the harmonic command from the subtraction of load current and output of the low-pass filter, this new algorithm does not affect the fluctuation of DC side capacitor voltage.

\section{Conflicts of Interest}

The authors declare that they have no conflicts of interest.

\section{Acknowledgments}

The authors would like to acknowledge financial support from National Key Research and Development Plan (no. 2016YFB0900204).

\section{References}

[1] B. Singh, S. Gairola, B. N. Singh, A. Chandra, and K. Al-Haddad, "Multipulse ac-dc converters for improving power quality: a review," IEEE Transactions on Power Electronics, vol. 23, no. 1, pp. 260-281, 2008.

[2] K. Z. Shuai, L. An, W. Zhu J et al., "Study on complex control of injection type hybrid active power filter," Proceedings of the Chinese Society of Electrical Engineering, vol. 28, no. 15, pp. 8491, 2008.

[3] P. Dang, T. Ellinger, and J. Petzoldt, "Dynamic interaction analysis of APF systems," IEEE Transactions on Industrial Electronics, vol. 61, no. 9, pp. 4467-4473, 2014.

[4] L. an, Harmonic Control and Reactive Power Compensation Technology and Equipment, China power press, Beijing, China, 2006.

[5] X. Xia, A. Luo, and S. Luo, "Application of high-capacity active power filter with single injection circuit," Electric Power Automation Equipment, vol. 30, no. 4, pp. 115-118, 2010.

[6] M. S. Hamad, M. I. Masoud, K. H. Ahmed, and B. W. Williams, "A shunt active power filter for a medium-voltage 12-pulse current source converter using open loop control compensation," IEEE Transactions on Industrial Electronics, vol. 61, no. 11, pp. 5840-5850, 2014.

[7] C. Gao, X. Jiang, and L. I. Yongdong, "A novel voltage selfbalance diode-clamped cascaded multilevel active power filter," Automation of Electric Power Systems, vol. 36, no. 12, pp. 94-100, 2012.

[8] X. Cui, C. Lam, W. Choi, and M. Wong, "Active part minimum capacity analysis of three-phase four-wire hybrid active power filters," Automation of Electric Power Systems, vol. 37, no. 3, pp. 122-128, 2013.

[9] C. Zhu, H. Yuan, and X. Guo, "Design and realization of harmonic extraction of active power filter based on DSP," Low Voltage Apparatus, 2012.

[10] A. Prasai and D. M. Divan, "Control of dynamic capacitor," IEEE Transactions on Industry Applications, vol. 47, no. 1, pp. 161-168, 2011.

[11] Q. Liu, Y. Deng, and X. He, "A novel AC-AC shunt active power filter without large energy storage elements," in Proceedings of the 14th European Conference on Power Electronics and Applications (EPE '11), pp. 1-9, IEEE, September 2011.

[12] C. Li, Y. Deng, Z. Lv, W. Li, X. He, and Y. Wang, "Virtual quadrature source-based sinusoidal modulation applied to high-frequency link converter enabling arbitrary direct AC-AC power conversion," IEEE Transactions on Power Electronics, vol. 29, no. 8, pp. 4195-4208, 2014.

[13] M. El-Habrouk, M. K. Darwish, and P. Mehta, "Active power filters: a review," Electric Power Applications, IEE Proceedings, vol. 147, no. 5, pp. 403-413, 2000.

[14] C. Yu, "Harmonic detection in electric power system based on wavelet multi-resolution analysis," in Proceedings of the International Conference on Computer Science and Software Engineering (CSSE '08), pp. 1204-1207, December 2008. 
[15] H. C. Lin, "Intelligent neural network-based fast power system harmonic detection," IEEE Transactions on Industrial Electronics, vol. 54, no. 1, pp. 43-52, 2007.

[16] M. Cirrincione, M. Pucci, G. Vitale, and A. Miraoui, "Current harmonic compensation by a single-phase shunt active power filter controlled by adaptive neural filtering," IEEE Transactions on Industrial Electronics, vol. 56, no. 8, pp. 3128-3143, 2009.

[17] F. Zhang, Z. Geng, and W. Yuan, "The algorithm of interpolating windowed FFT for harmonic analysis of electric power system," IEEE Transactions on Power Delivery, vol. 16, no. 2, pp. 160-164, 2001.

[18] M. K. Darwish, "Design and implementation of a modified fourier analysis harmonic current computation technique for power active filters using DSPs," IEEE. Electrical Power Application, vol. 148, no. 1, pp. 21-28, 2001.

[19] W. M. Grady, A. H. Noyola, and M. J. Samotyj, "Survey of active power line conditioning methodologies," IEEE Transactions on Power Delivery, vol. 5, no. 3, pp. 1536-1542, 1990.

[20] L.-Q. Tong, Z.-M. Qian, and F.-Z. Peng, "Synchronous reference frame harmonic detection modeling and digital realization," Proceedings of the Chinese Society of Electrical Engineering, vol. 29, no. 19, pp. 111-117, 2009.

[21] N. F. A. Rahman, M. K. Hamzah, S. Z. M. Noor, and A. S. A. Hasim, "Single-phase hybrid active power filter using single switch parallel active filter and simple passive filter," in Proceedings of the International Conference on Power Electronics and Drive Systems (PEDS '09), pp. 40-45, IEEE, Taipei, Taiwan, January 2009.

[22] L. Asiminoaei, F. Blaabjerg, and S. Hansen, "Evaluation of harmonic detection methods for active power filter applications," in Proceedings of the 20th Annual IEEE Applied Power Electronics Conference and Exposition (APEC '05), pp. 635-641, March 2005.

[23] P. Zheng, P. Wang, Y. Sui, C. Tong, F. Wu, and T. Li, "Near-fivevector SVPWM algorithm for five-phase six-leg inverters under unbalanced load conditions," Journal of Power Electronics, vol. 14, no. 1, pp. 61-73, 2014.

[24] C.-S. Lam, M.-C. Wong, W.-H. Choi, X.-X. Cui, H.-M. Mei, and J.-Z. Liu, "Design and performance of an adaptive lowDC-voltage-controlled LC-hybrid active power filter with a neutral inductor in three-phase four-wire power systems," IEEE Transactions on Industrial Electronics, vol. 61, no. 6, pp. 26352647, 2014.

[25] L. Asiminoael, F. Blaabjerg, and S. Hansen, "Detection is key-harmonic detection methods for active power filter applications," IEEE Industry Applications Magazine, vol. 13, no. 4, pp. 22-33, 2007.

[26] Y. F. Wang and Y. W. Li, "An overview of grid fundamental and harmonic components detection techniques," in Proceedings of the 5th Annual IEEE Energy Conversion Congress and Exhibition (ECCE '13), pp. 5185-5192, September 2013.

[27] C. Li, Y. Deng, H. Peng, X. He, W. Li, and Y. Wang, "Partial power conversion device without large electrolytic capacitors for power flow control and voltage compensation," IEEE Transactions on Power Electronics, vol. 27, no. 12, pp. 4847-4857, 2012.

[28] H. Akagi, E. H. Watanabe, and M. Aredes, "Instantaneous Power Theory and Applications to Power Conditioning," Instantaneous Power Theory and Applications to Power Conditioning, pp. 1-379, 2006. 


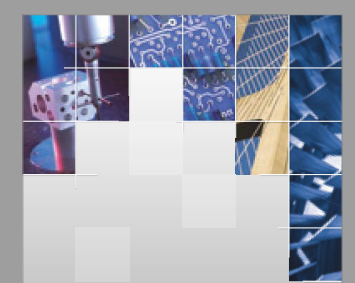

\section{Enfincering}
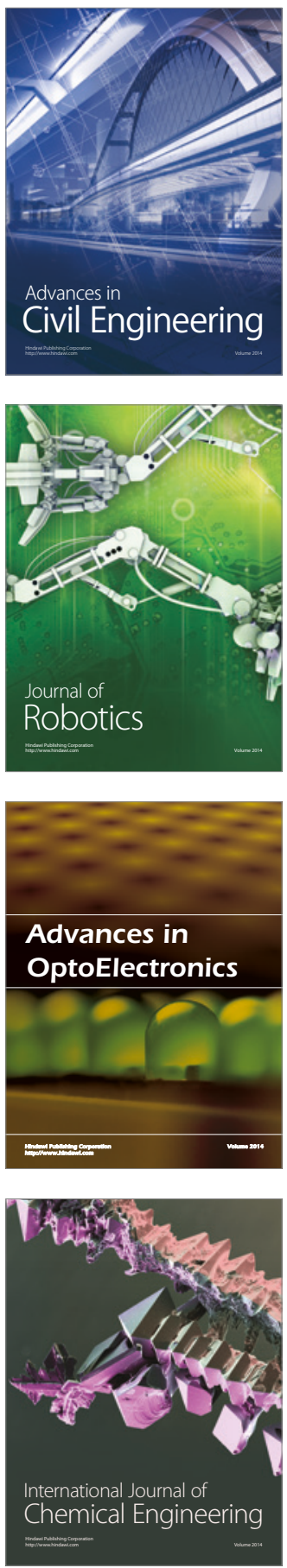

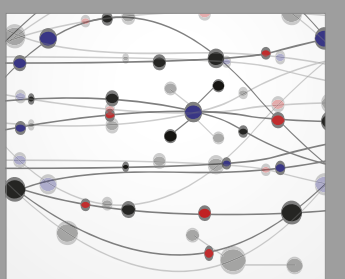

The Scientific World Journal

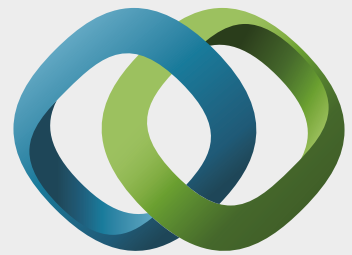

\section{Hindawi}

Submit your manuscripts at

https://www.hindawi.com
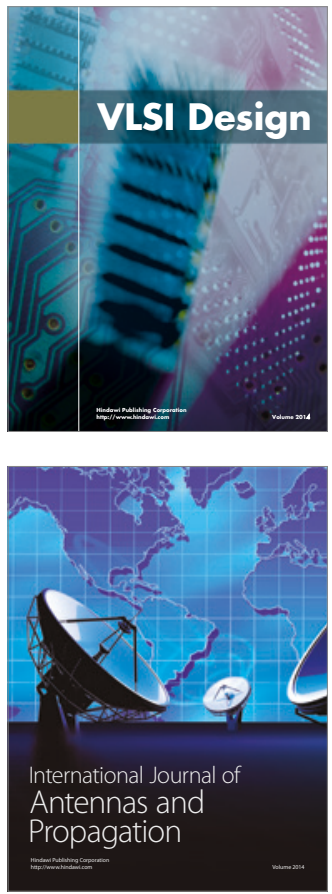

\section{Rotating}

Machinery
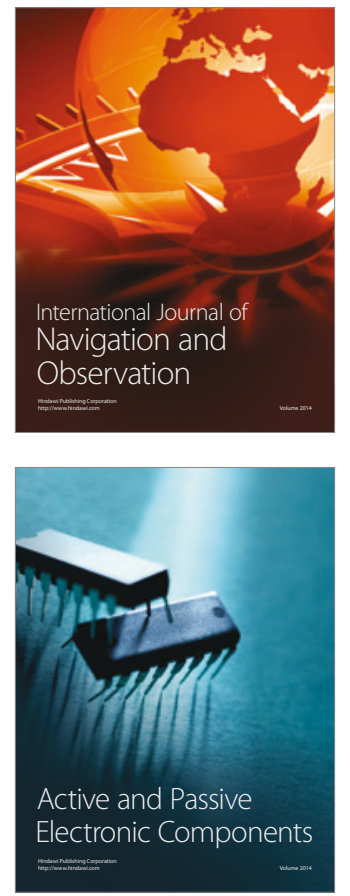
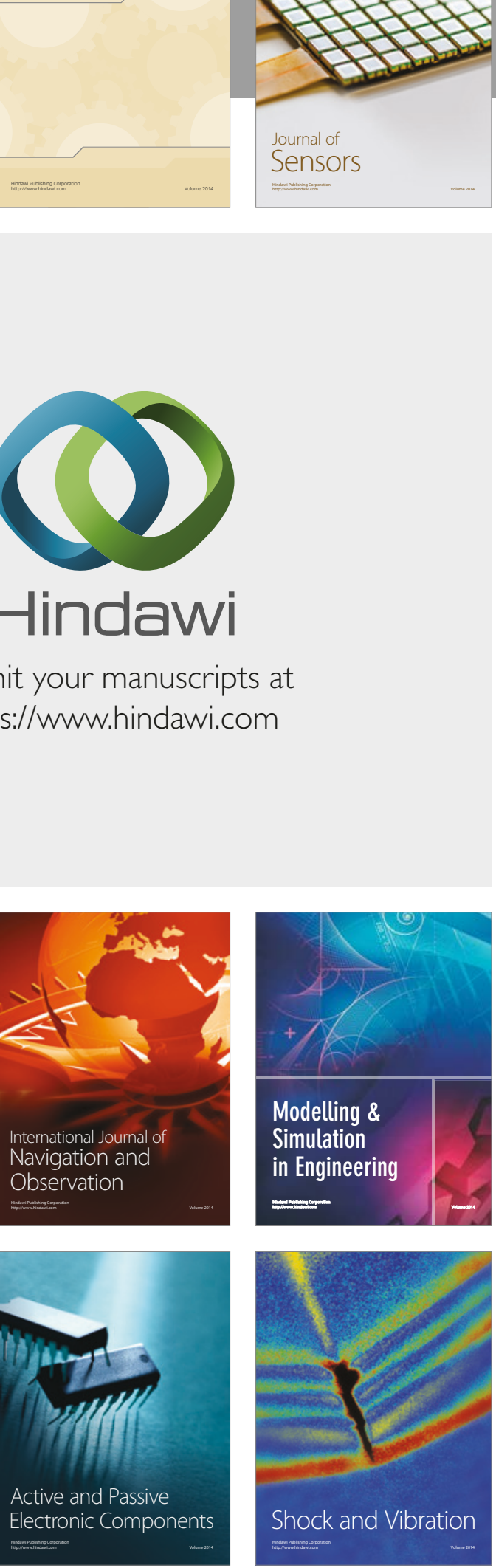
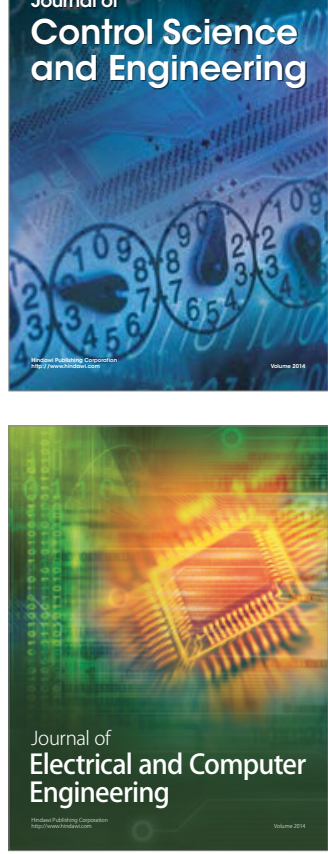

Distributed

Journal of

Control Science

and Engineering
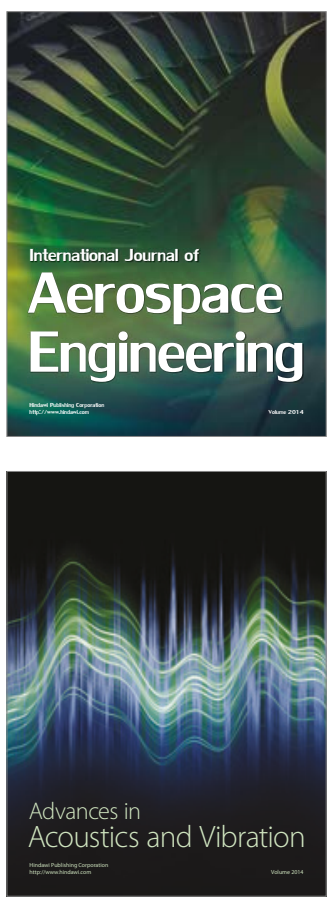

Sensor Networks 